$$
\begin{aligned}
& \text { تقليل توافقيات التيار مع تحسين عامل القدرة للمقومات أحادية الطور المحكومة بتقتية } \\
& \text { حقن ثو افقيات التيار } \\
& \text { أ.د.باسل محمد سعيد الكربائية/جامعة الموصين ابزار زينل }
\end{aligned}
$$

الخلاصة

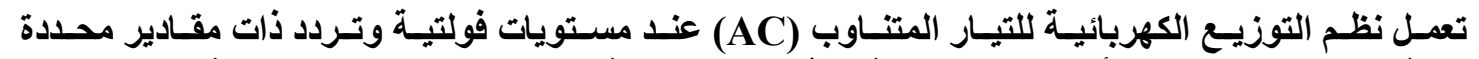

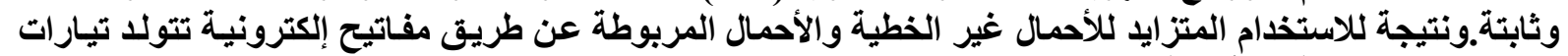



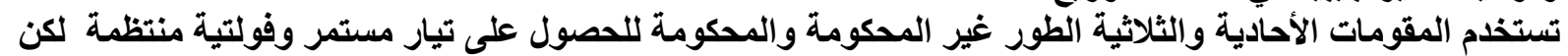

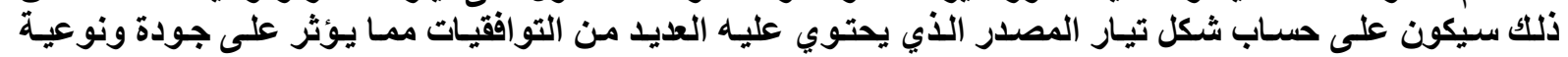

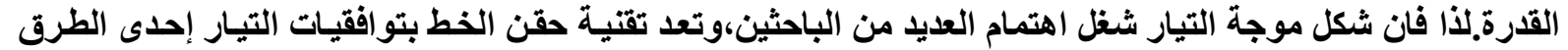

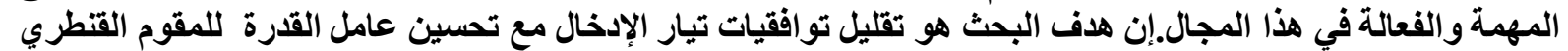

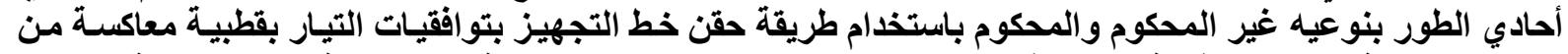

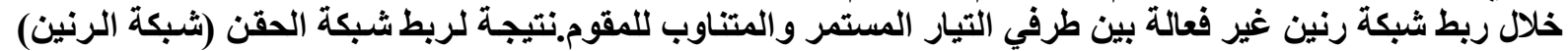

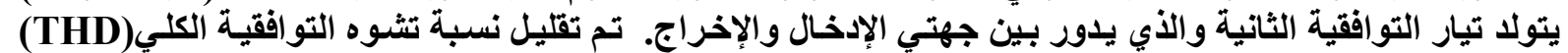

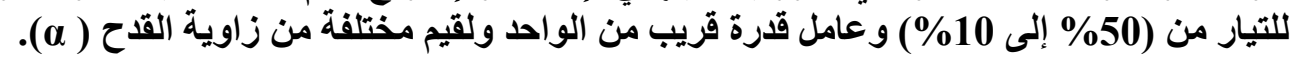

\title{
AC Current Harmonics Reduction\& improve the power factor for Single Phase controlled Rectifiers Using Current Injection Method
}

\section{Basil M.Saied}

Ph. D.

\section{Hussein I.Zynal}

Ph. D.

Dept. of Electrical Engineering, Mosul University

\author{
Aasef.A.Saleh \\ M.Sc.
}

\section{Abstract}

The AC electrical distribution system works at limited values of voltage and frequency levels. The increase of using non linear loads and loads those connected by electronic keys produces non sinusoidal voltages and currents in the distribution systems.

Single and three phase, controlled and uncontrolled rectifiers, are used for obtaining DC current and voltage. This affect the shape of the supply current which included many harmonics. The quality and quantity of the power obtained are affected as a result. The shape of the current wave is therefore attracted the attention of many researcher groups. The line injection by current harmonics is one of the most important and effective techniques used for such treatment.

The aim of this research work is to reduce the harmonics in the input current and improving the power factor of the single phase, controlled and uncontrolled, rectifiers. This is usually carried out by employing injection method technique with reversible polarity through connecting a passive resonance circuit between the input and output of the rectifier. The passive resonance circuit creates second harmonic current which will circulate between the AC and DC sides of the rectifier and decreases the total harmonic distortion (THD). In this work, the ratio of (THD) is reduced from $50 \%$ to $10 \%$ with power factor increased nearly to unity for different values of the trigger angle $(\alpha)$. 


\begin{tabular}{|c|c|}
\hline \multicolumn{2}{|l|}{ قائمة الرموز: } \\
\hline القيمة المؤثرة لمركبات التو افقيات لتبار الإدخال بعد الحقن = In(n) & f = تردد المصدر \\
\hline القيمة المؤثرة لتيار الإدخال قبل الحقن = In & f = f $=f_{2}$ \\
\hline = = Im & م = عامل الحقن \\
\hline قيمة الذروة لتبار التو افقية الثانية المار في محاثة الضبط $=I_{f m}$ & 年 $=\alpha$ \\
\hline القيمة المؤثرة لتيار التو افقية الثنانية المار في محاثة الضبط = If & القيمة الآنية لتيار الإدخال قبل الحقن $=i_{\text {in }}$ \\
\hline v = القيمة الآنية لفولتية إدخال المقوم & القيمة الآنية لتيار الإدخال بعد الحقن =in \\
\hline قيمة الآنبة لفولتية التوافقية الثانية & 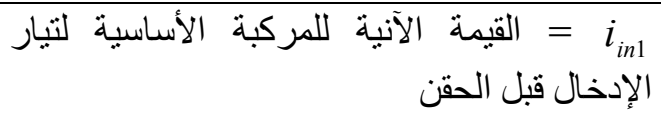 \\
\hline ق قيمة الذروة لفولتية إدخال المقوم = V & القinj = القيمة الآنية لتيار الحقن المثالي \\
\hline القيمة المؤثرة لفولتية دائرة ثقنن (مركبة التو افقية الثانية) & القيمة = الآنية لتيار الحقن الفعلي \\
\hline ق قيمة الذروة لفولتية دائرة ثفنن & ملف الضبط $=i_{f}$ \\
\hline 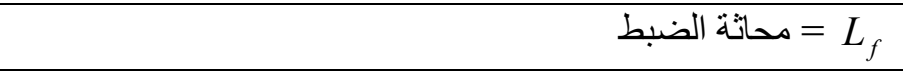 & 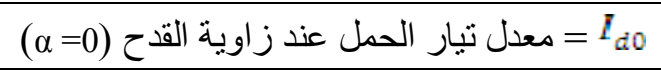 \\
\hline 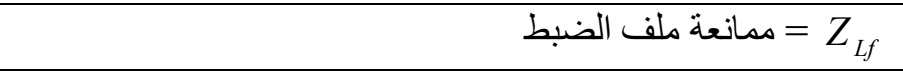 & 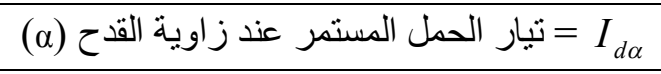 \\
\hline & 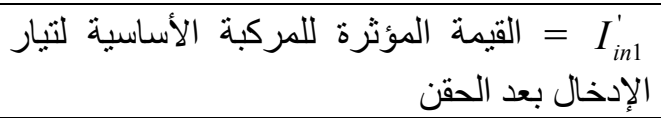 \\
\hline
\end{tabular}



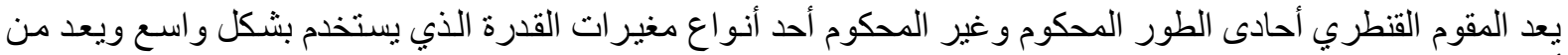

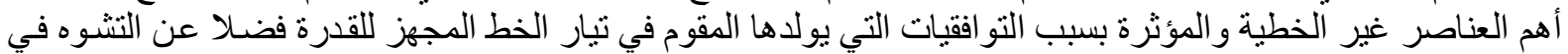



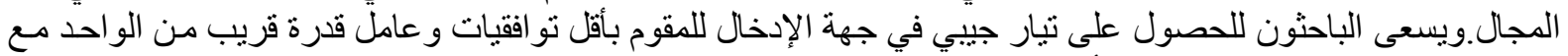

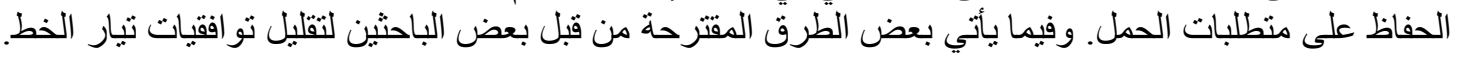

قام الباحثون (Atluri Rama Prasad , Phoivos D.Ziogas and Stefanos Manias) باقتر اح طريقة جديدة لتقليل

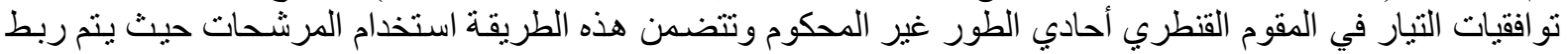

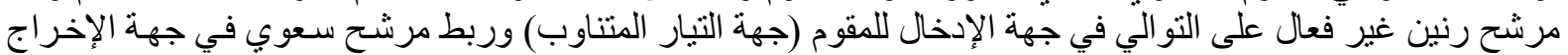

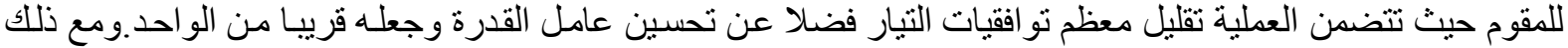

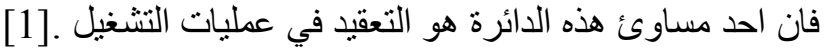

كذللك اقترح الباحثان (S.Kim,P.Enjeti) طريقة جديدة لتحسين عامل القدرة واختز ال تو افقيات التيار للمقوم القنطري ثلاثني

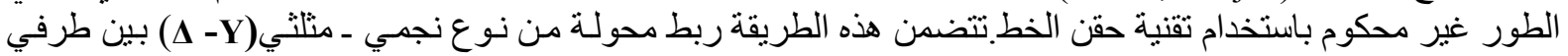



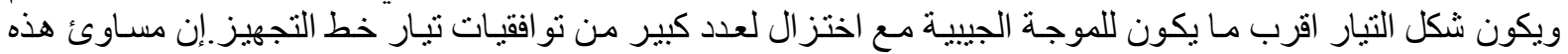

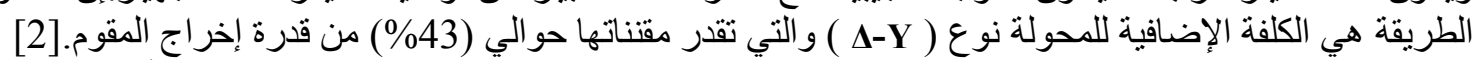

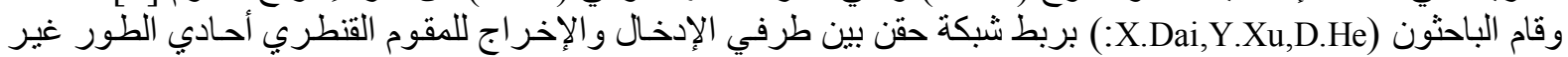



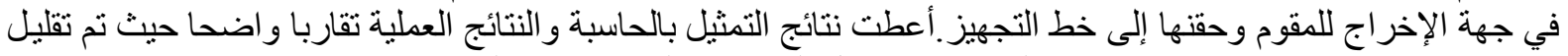

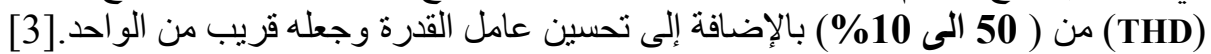

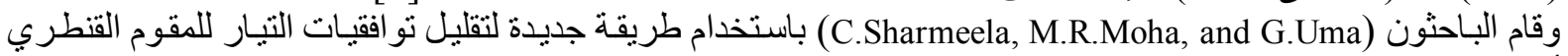




خلال المرشح الحثي وجعله بنفس الطور مع تيار الإدخال فضـلا عن تحسين شكل موجـة التيار وقد أعطت هذه الطريقة الطية 




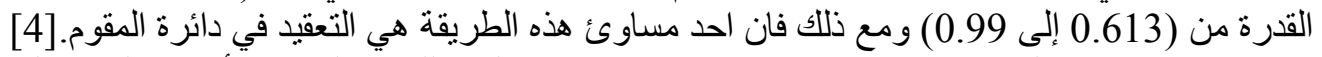

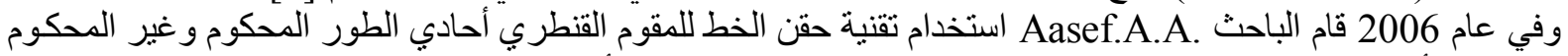

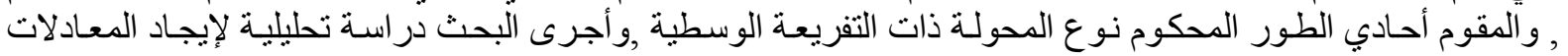

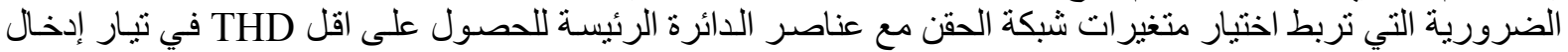



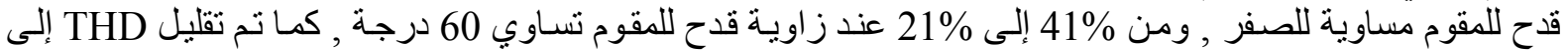

13\% عند استخدام المحولة ذات التفريعة الوسطية. اقترح الباحثان Basil M.Saied and Hussein I.Zynal طريقة جديدة لتقليل تو افقيات التيار وتحسين عامل القدرة



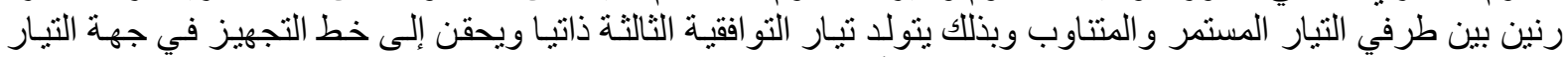

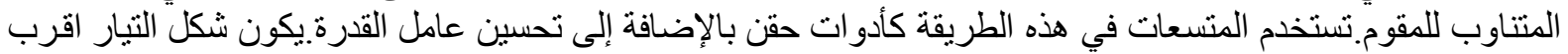



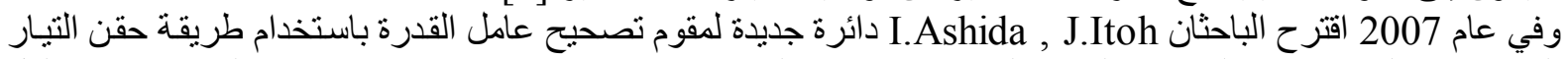

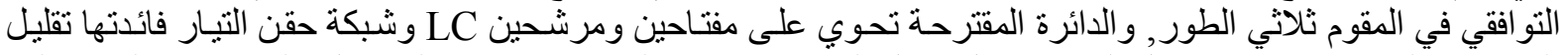

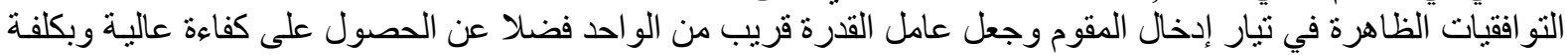

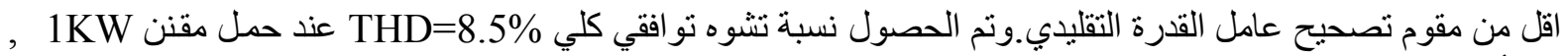

كما أنها تخفض خسائر المقوم بمقدار

2- وصف الأئرة المقترحة: من خلال ماجاء في أعلاه للمقوم أحادي الطورة الطور نلاحظ أن معظم الطرق المستخدمة مع المقوم أحادي الطور في حالة المقوم

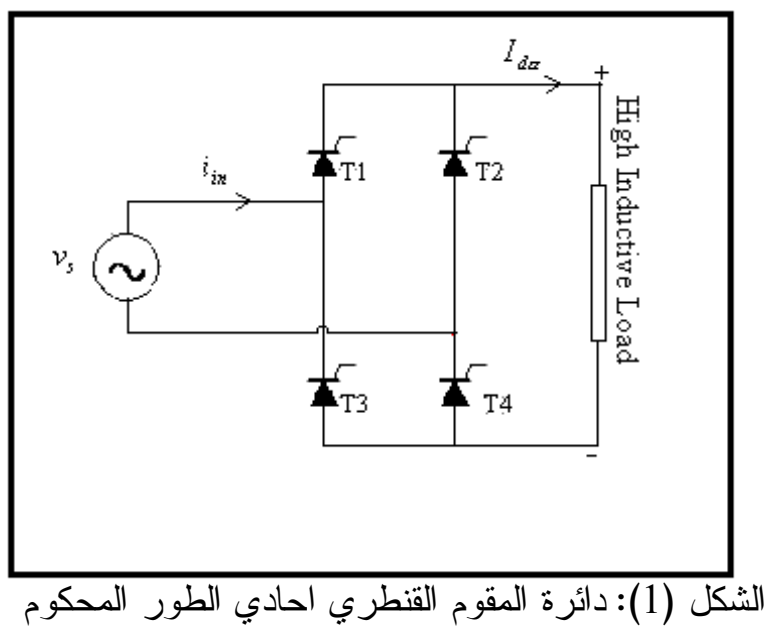

مع شبكة الحقن

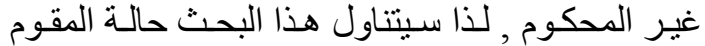





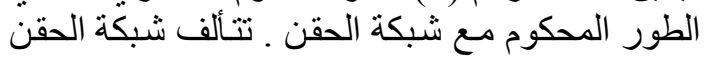

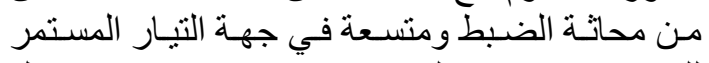

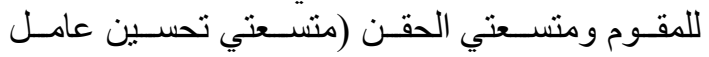
القدرة)في جهة التيار المتناوب.

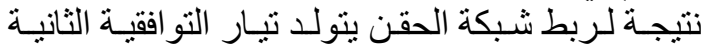

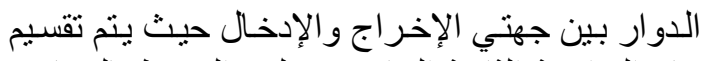

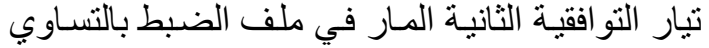

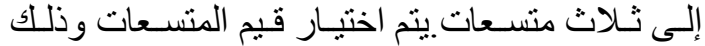

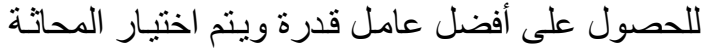
لضبط قيمة وطور نتار التو افقية الثانية.

\section{3- توليد فولتية التو افقية الثانية ذاتيا:}



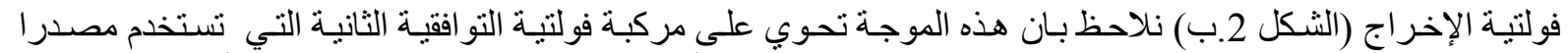



التجهيز هي: تيار التيا

$v_{s}=V_{m} \sin w t$

عند تحليل الموجة في الثكل(2.ب) باستخدام منو الية فورير يمكن كتابة معادلة مركبة التو افقية الثانية للفولتية كالاتي: $v_{2}(w t)=-\frac{2 V_{m}}{\sqrt{3} \pi}\left(\frac{10}{3}-2 \cos 2 \alpha\right)^{\frac{1}{2}} \sin \left(2 w t+\gamma_{2}\right)$

حيث أن ( $\left.\gamma_{2}\right)$ هي الزاوية بين فولتية التوافقية الثانية والفولتية الأساسية. 


$$
\gamma_{2}=\tan ^{-1} \frac{\left(\frac{2}{3} \cos 3 \alpha-2 \cos \alpha\right)}{\left(\frac{2}{3} \sin 3 \alpha-2 \sin \alpha\right)}
$$

يتضح من المعادلتين (2,3) بان قيمة وطور مركبة فولتية التو افقية الثانية يعتمد على زاوية القدح (a).

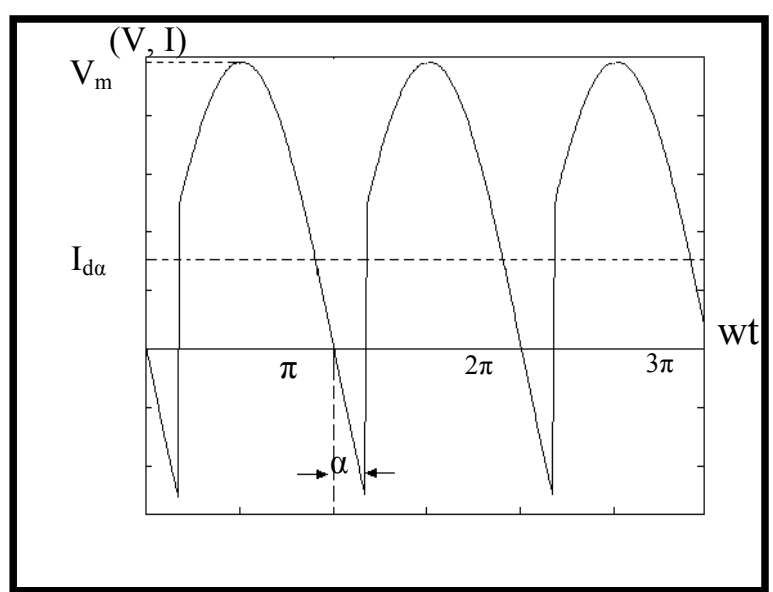

(ب)

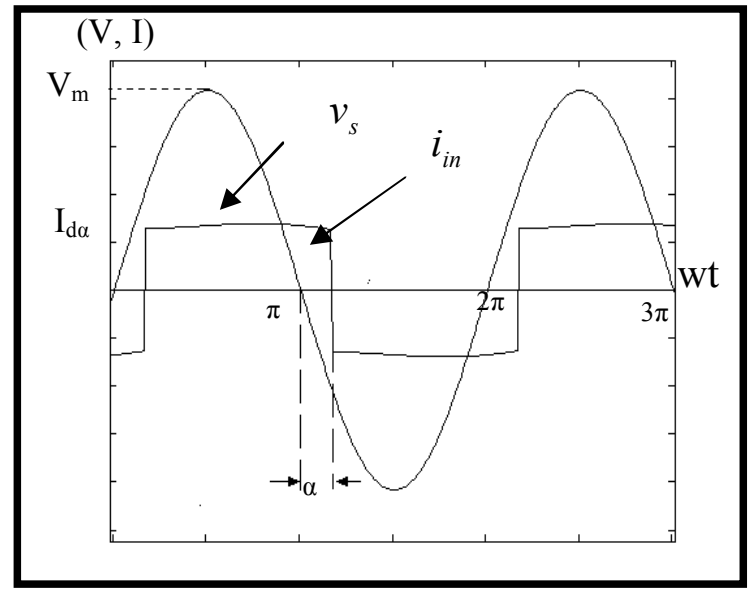

(ا)

الثكل (2):موجة فولتية وتيار الإدخال و الإخر اج للمقوم

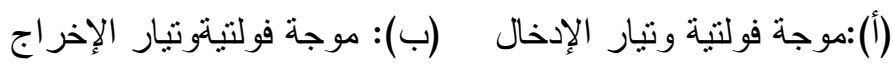

4- تحليل تيار الإدخال:

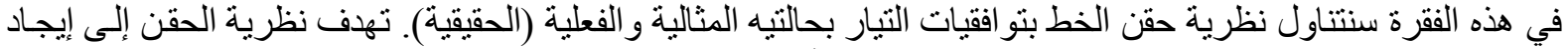

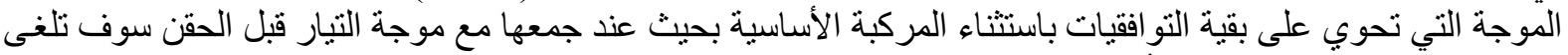
التو افقيات وتنقى مركبة التيار الأساسية فقط بلتئ.

1-4: تحليل مبأ الحقن من الناحية النظرية النية (الحالة المثالية)

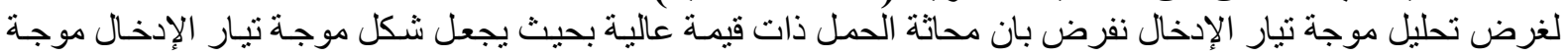

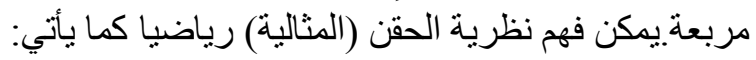
$i_{i n}=i_{i n 1}+i_{i n j}$

حيث يمثل (

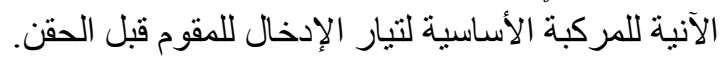

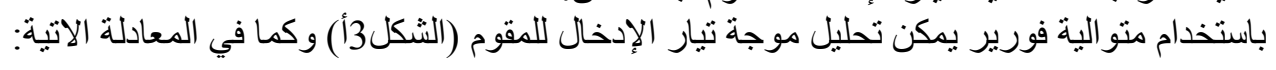

$i_{\text {in }}=I_{d \alpha}[1.27 \sin (w t-\alpha)+0.4244 \sin 3(w t-\alpha)+0.2546 \sin 5(w t-\alpha)$

$$
+0.1818 * \sin 7(w t-\alpha)+\ldots]
$$

لذا يمكن التعبير عن تيار الحقن و المبين في الثكل (3.ب) بالمعادلة الآتية:

$\therefore i_{i n j}=-I_{d \alpha}[0.4244 \sin 3(w t-\alpha)+0.2546 \sin 5(w t-\alpha)+0.1818 \sin 7(w t-\alpha)+\ldots]$

نلاحظ من المعادلة (6) إن تبار الحقن هو عبارة عن المنو الية التي تشمل كل مركبات التو افقيات لتيار الإدخال و عند جمعها



$i_{i n}^{\prime}=1.27 * I_{d \alpha} * \sin (w t-\alpha)$ 
حيث يمثل (in ) القيمة الآنية لتيار الإدخال للمقوم بعد الحقن.

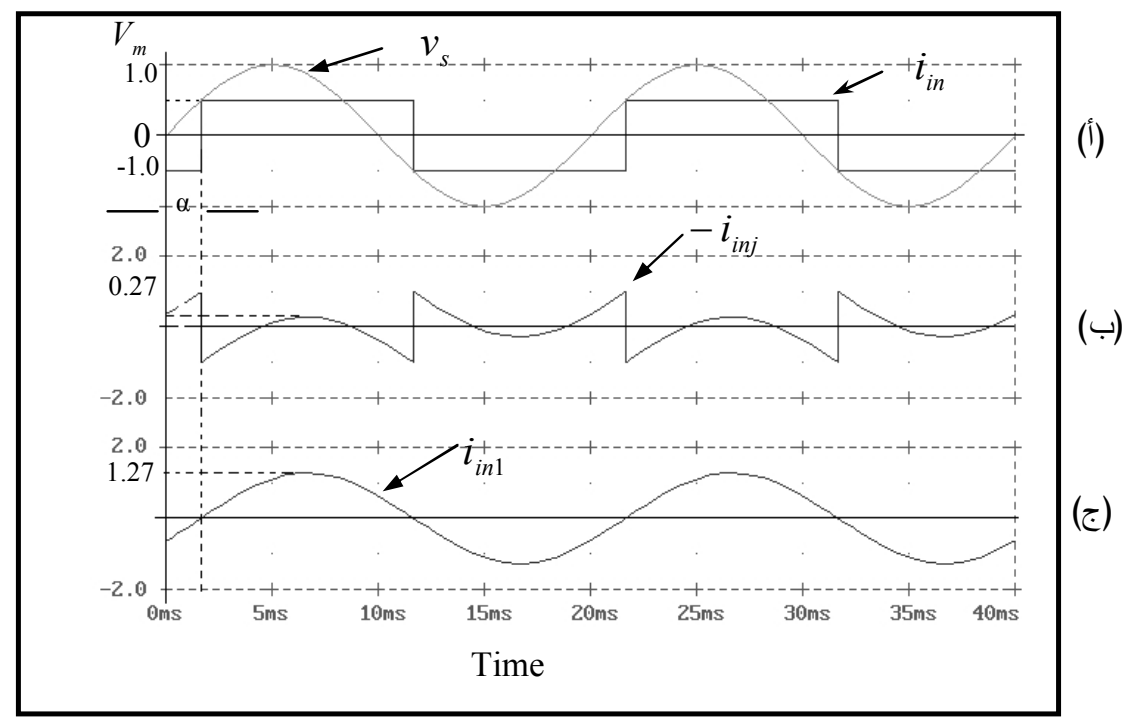

الشكل (3): موجات التيار (الحالة المثالية) (أ):موجة تيار الإدخال قبل الحقن (ب): موجة تيار الحقن (ج): موجة المركبة الأساسية لتيار الإدخال (تيار الإدخال

2-4 تحليل نظرية الحقن من الناحية الفعلية(الحقيقية)

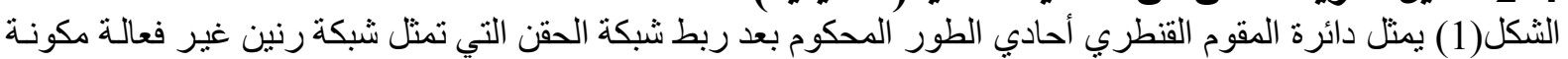

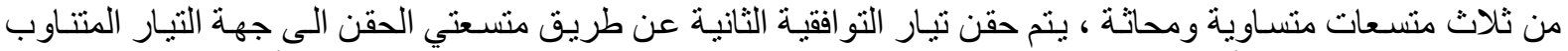

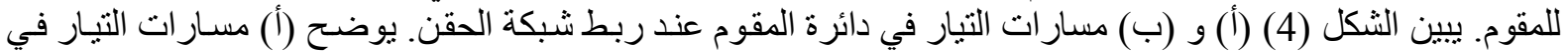


في حالة توصيل.يتضح من الثكل بان تيار المصدر بعد الحقن يساوي (T $\left(T_{3} 6 T_{2}\right)$ لتيار التو افقية الثانية.

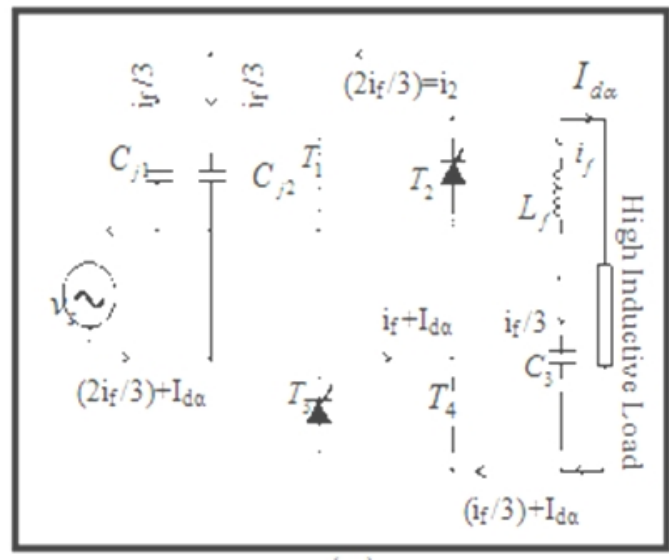

$(ب)$

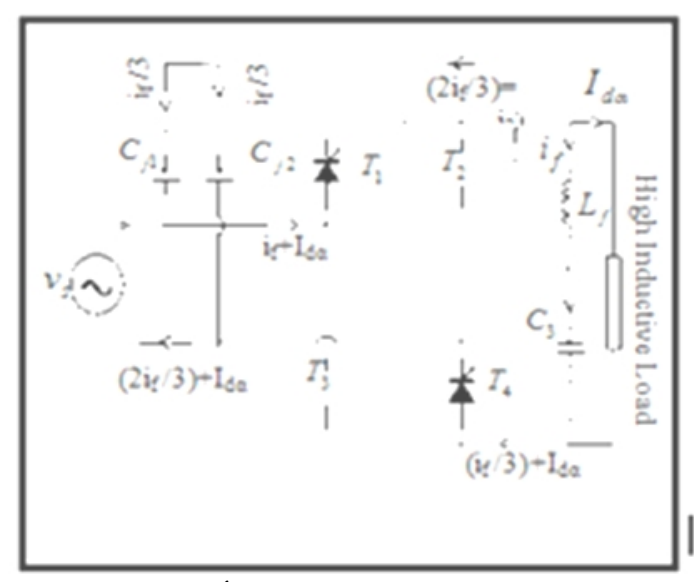

(ا)

الثكل (4): نوضيح مسار ات التبار ات لدائرة المقوم القنطري أحادي الطور المحكوم مع شبكة الحقن (أ):( )

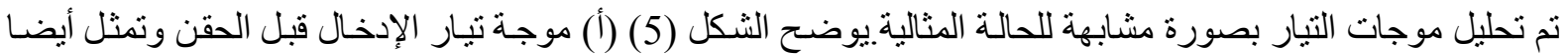

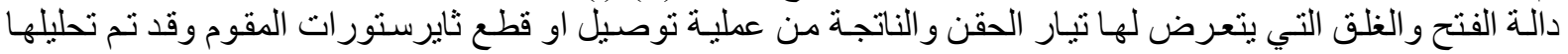


بوساطة متو الية فورير والتي تم بيانها في المعادلة (6).وتمثل الموجة في (ب) من هذا الثكل تيار التوافقية الثنانية ( وهو جزء من تبار ملف الضبط ( يمكن التعبير عن ( )

$$
i_{2}=-I_{m} \cos 2(w t-\alpha)=-I_{m} \sin \left(2 w t-2 \alpha+\frac{\pi}{2}\right)
$$

حيث أن ( ${ }$ ) هي قيمة الذروة لتيار التو افقية الثنانية. يمكن تعريف عامل الحقن رياضياً بأنه النسبة بين قيمة الذروة التيار التوارة التقية الثانية الى قيمة الذروة لتيار الحمل

$$
\rho=\frac{I_{m}}{I_{d \alpha}}
$$

و عند حقن التيار الى جهة الإدخال للمقوم عن طريق منسعتي الحقن يتعرض الى عملية الفتح والغلق وكما مبين في المعادلة

$i_{i n j}^{\prime}=i_{2}=-\rho * I_{d \alpha} * \cos 2(w t-\alpha)$

$i_{i n j}^{\prime}=-i_{2}=\rho^{*} I_{d \alpha} * \cos 2(w t-\alpha)$

$$
\begin{gathered}
(\alpha<w t<\pi+\alpha) \\
(\pi+\alpha<w t<2 \pi+\alpha)
\end{gathered}
$$

حيث يمثل ( ${ }^{\prime}$ ) تيار الحقن الفعلي.ييين الفرع (ج) من الثكل (5) موجة تيار الحقن الفعلي بعد عمليتي الفتح و الغلق لذا بمكن كتابة معادلة تبار الحقن كما يأتي :-

$i_{i n j}^{\prime}=S W * i_{2}$

$i_{i n j}^{\prime}=S W^{*}\left(-\rho * I_{d \alpha} * \cos 2(w t-\alpha)\right)$

حيث تمثل (SW) دالة الفتح و الغلق و المبين خصائصها في الملحق (B) (B) ).

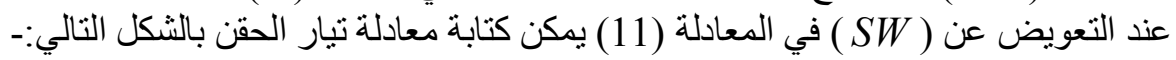
$i_{i n j}^{\prime}=\rho I_{d \alpha}[0.427 \sin (w t-\alpha)-0.765 \sin 3(w t-\alpha)-0.303 \sin 5(w t-\alpha)-$

$0.198 \sin 7(w t-\alpha)+\ldots]$

وللحصول على موجة التيار بعد الحقن يتم جمع موجة التيار قبل الحقن في الشكل (5) (أ) مع تبار الحقن الفعلي في الشكل

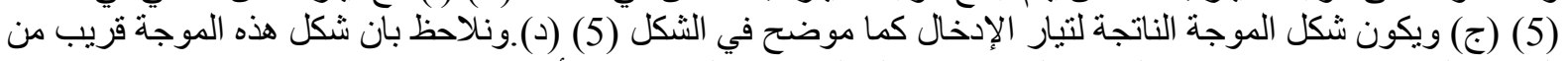
الموجة الجيبية ويمكن كتابة معادلة هذه الموجة (معادلة الإدلة التيار بعد الحقن) كما يأتي:-

$$
i_{i n}^{\prime}=i_{i n}+\left(S W^{*} i_{2}\right)=i_{i n}+i_{i n j}^{\prime}
$$

و عند التعويض عن (in $i_{i n}$ من المعادلة (5) و(' ${ }^{\prime}$ (inj ) من المعادلة (12) في المعادلة (13) ينتج:$i_{\text {in }}^{\prime}=I_{d \alpha}[(1.27+0.427 \rho) \sin (w t-\alpha)+(0.4244-0.765 \rho) \sin 3(w t-\alpha)$

$$
\begin{aligned}
& +(0.2546-0.303 \rho) \sin 5(w t-\alpha)+(0.1818-0.198 \rho) \sin 7(w t-\alpha) \\
& +(0.144-0.148 \rho) \sin 9(w t-\alpha)+\ldots]
\end{aligned}
$$

المعادلة(14) تبين بأن قيمة الذروة لكل نو افقية في تبار الإدخال بعد الحقن تعتمد على قيمة الذروة لتيار التو افقبة الثانية

$$
\text { . }\left(I_{m}=\rho I_{d \alpha}\right)
$$




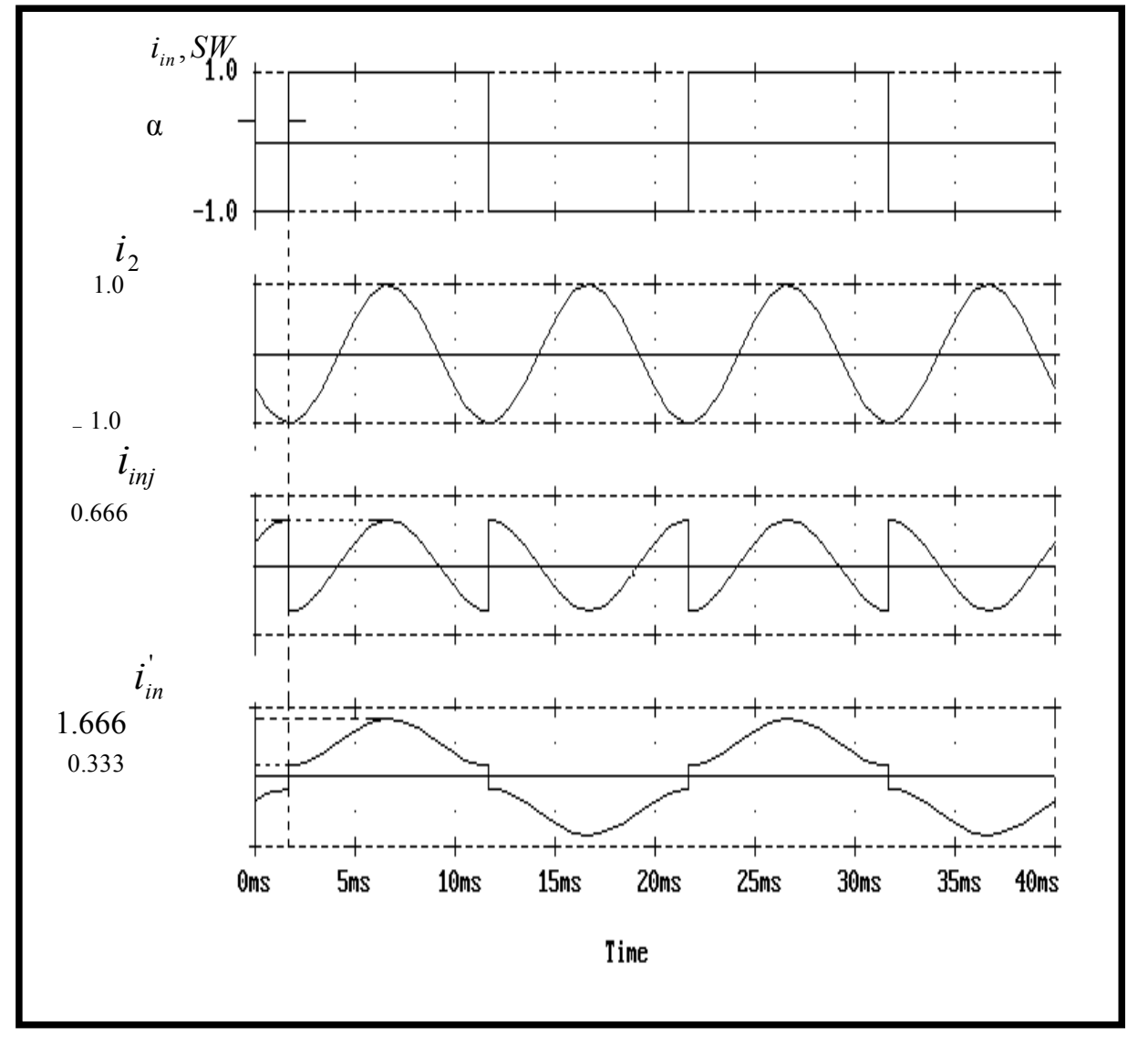

(أ)

$(ب)$

(ج)

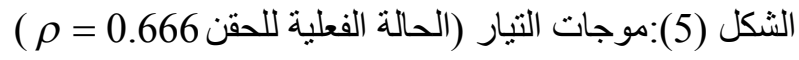

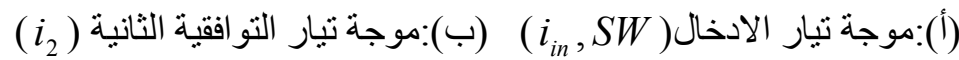

(ج):موجة تيار الحقن(

ولمقارنة الحالة الفعلية للحقن مع الحالة المثالية نلاحظ أن الثكل (6) الفرع ع (أ) يبين موجة تبار الحقن المثالية مع الطيف

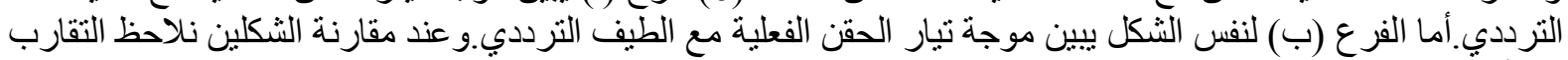

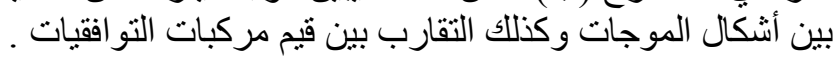

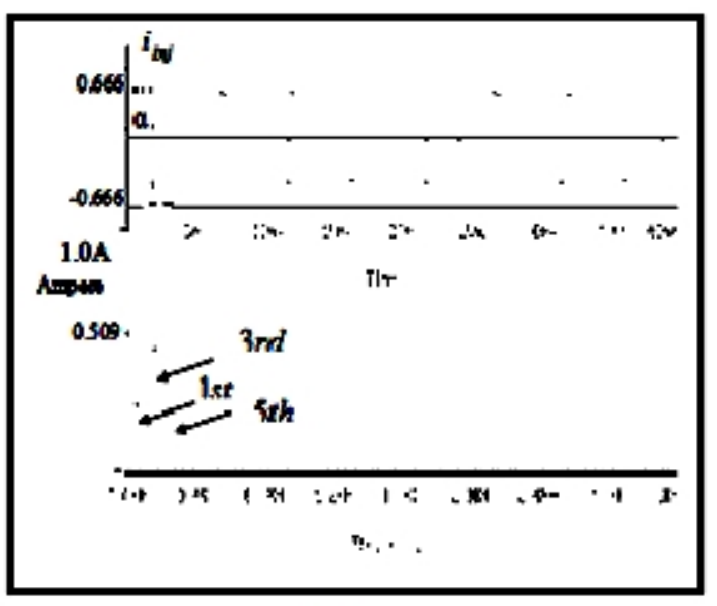

(4)

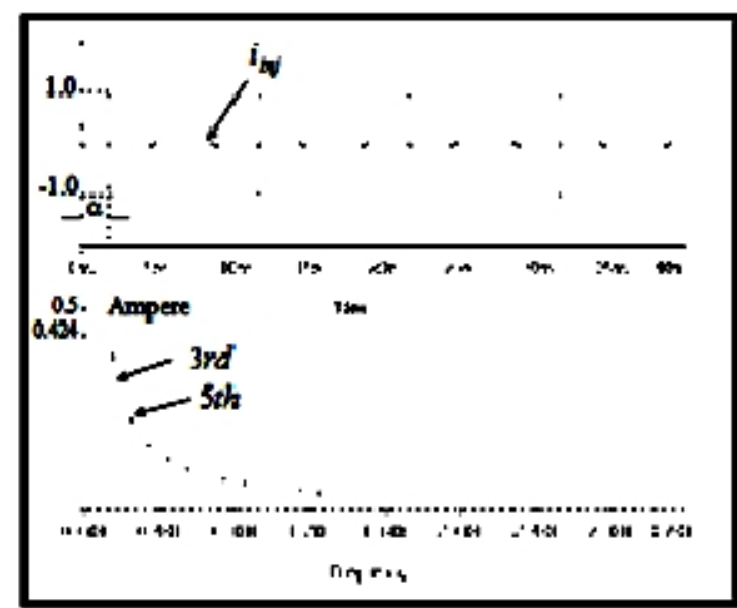

(i)

الشكل (6):موجة تيار الحقن مع الطيف الترددي ( 0.666 = م) (أ):الحالة المثالية (ب):الحالة الفعلية 


\section{5-إيجاد أفضل عامل حقن (}

يمكن تعريف عامل النوعية و الذي يسمى تشوه النو افولقية الكلية للتبار حسب المعادلة الآتية:

$T H D=\sqrt{\frac{I_{i n}^{2^{\prime}}-I_{i n 1}^{2^{\prime}}}{I_{i n 1}^{2^{\prime}}}}$

بالتعويض عن قيم مركبات تو افقيات التيار من المعادلة (14) في المعادلة (15) ينتج:

$T H D=\frac{1}{0.427 \rho+1.27} \sqrt{0.714 \rho^{2}-0.88 \rho+0.278}$

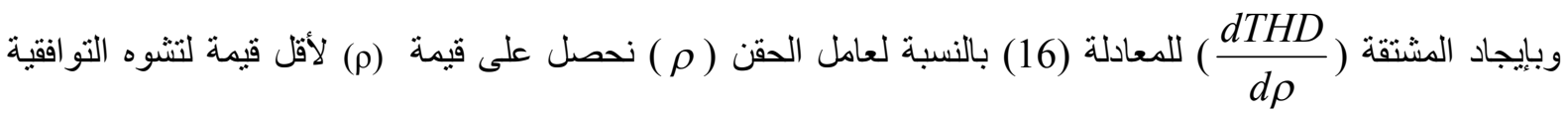

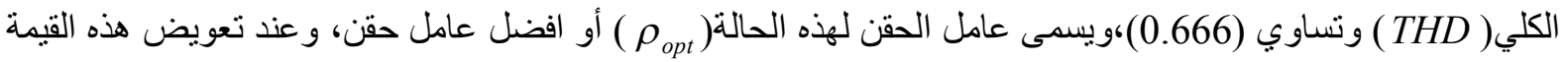
في المعادلة (16) تكون قيمة (THD) (THD) مساوية تقريبأ (10.666).



$i_{i n}^{\prime}=I_{d \alpha}[1.556 \sin (w t-\alpha)+0.082 \sin 3(w t-\alpha)+0.055 \sin 5(w t-\alpha)+$

$0.051 \sin 7(w t-\alpha)+0.045 \sin 9(w t-\alpha)+\ldots]$



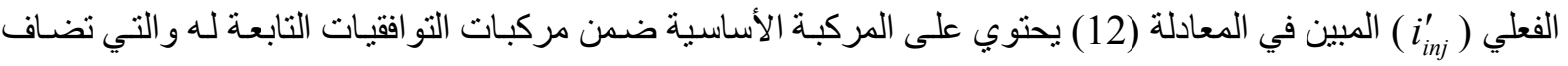

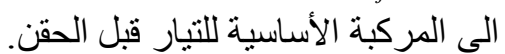
إن قيمة النروة لمركبات التوافقيات لتيار الإدخال بعد الحقن قد قلت بشكل كبير مما يدل على فعالية عملية الحقن.

6- إيجاد قيمة وطور تيار التوافقية الثانية للحصول على تيار التواوقية الثانية ذاتيًا يجب أن تكون دائرة الحقن في حالة رنين (أو شبه رنين) لتنوير هذا التبار بين



الثكل(7):مخطط دائرة تفنن المكافئة بين (A,B) للائرة في الشكل (1)

$$
\begin{aligned}
& Z_{t h}=R_{t h}+j X_{t h} \\
& X_{t h}=\frac{1}{2 \pi f_{2}\left(C_{j 1}+C_{j 2}+C_{3}\right)}
\end{aligned}
$$
بالرجوع الى الثكل(1) نلاحظة بان دائرة

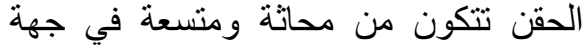
الإخراج ومن متسعتي الحقن في جهة فية

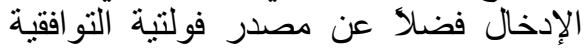

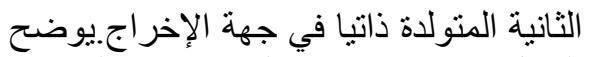
الثكل (7) دائرة ثقنن المكافئة بين النقطتين للائرة الميبنة في الثكل (1) (B,A) $V_{t h}^{\wedge}=V_{2}=\frac{2 V_{m}}{\sqrt{3} \pi}\left(\frac{10}{3}-2 \cos 2 \alpha\right)^{\frac{1}{2}}$

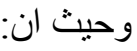

يمكن ايجاد قيمة النزوة لتنار التو افقية الثانية من الثكل (7) وكما يأتي: 


$$
I_{f m}=\frac{V_{t h}^{\wedge}}{\left|Z_{t h}+Z_{L f}\right|}=\frac{2 V_{m}}{\sqrt{3} \pi\left|Z_{t h}+Z_{L f}\right|}\left(\frac{10}{3}-2 \cos 2 \alpha\right)^{\frac{1}{2}}
$$

وللحصول على افضل حالة حقن يجب ضبط قيمة وطور تيار التوافقية الثانية وكما يلي: أـ القيمة العظمى لتيار التو افقية الثانية (

$$
I_{m}=\frac{2}{3} I_{f m}
$$

$$
I_{f m}=I_{d \alpha}
$$

من المعادلتين (9) و (22) و لأفضل حالة حقن ( 1 (23

$$
\left|Z_{t h}+Z_{L f}\right|=\frac{2 V_{m}}{\sqrt{3} \pi I_{d \alpha}}\left(\frac{10}{3}-2 \cos 2 \alpha\right)^{\frac{1}{2}}
$$

حيث أن

من المعادلتين (21) و (23) يمكن حساب |.

بـ في الثكل (7) يمكن كتابة معادلة التيار الآني بالصيغة الآتية: ... (25) $i_{f}(w t)=-I_{f m} \sin \left(2 w t+\gamma_{2}-2 \beta_{2}\right)$ ( $\left.\beta_{2}\right)$ هي الزاوية بين فولتية التو افقية الثانية وتيار التوافقية الثانية وتساوي: (25)

$$
\beta_{2}=\cos ^{-1}\left[\frac{R_{t h}+R_{L f}}{\left|Z_{t h}+Z_{L f}\right|}\right]=\sin ^{-1}\left[\frac{X_{t h}-X_{L f}}{\left|Z_{t h}+Z_{L f}\right|}\right]
$$

وبما أن ( $)$

$i_{2}(w t)=-I_{m} \sin \left(2 w t+\gamma_{2}-2 \beta_{2}\right)$

وللحصول على أفضل طور لتيار التو افقية الثانية يجب أن يتفق طور تنيار التو اققية الثانية مع طور المركبة الأساسية لتبار

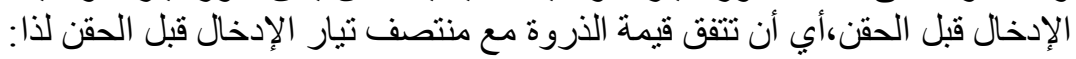

$2 w t-2 \alpha+\frac{\pi}{2}=2 w t+\gamma_{2}-2 \beta_{2}$

$\beta_{2}=\frac{\gamma_{2}}{2}+\alpha-\frac{\pi}{4}$

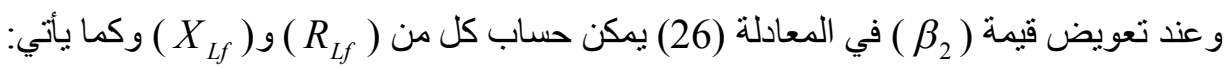

$R_{L f}=\left|Z_{t h}+Z_{L f}\right| \cos \beta_{2}-R_{t h}$

$X_{L f}=\left|Z_{t h}+Z_{L}\right| \sin \beta_{2}-X_{t h}$

$L_{f}=\frac{X_{L f}}{2 \pi f_{2}}$

لذا يتم حساب (

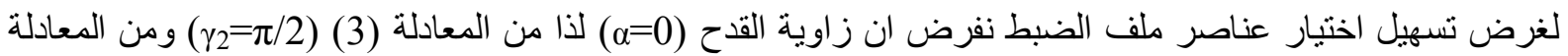


رنين لذا ( ( ) بالف بالنقصان ولضبط قيمة وطور تيار التو افقية الثانية يجب زيادة قيمة محاثة ملف الضبط. 
7-نتائج تمثيل دائرة المقوم القنطري

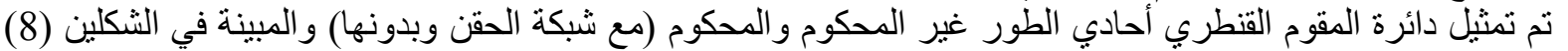



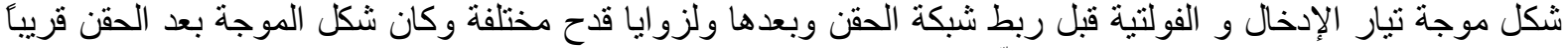


الحقن وبعدها ويبين الملحق (A) المعادلات الخاصة بالحساب النظري لكل من (U) مع دائرة التمثيل.

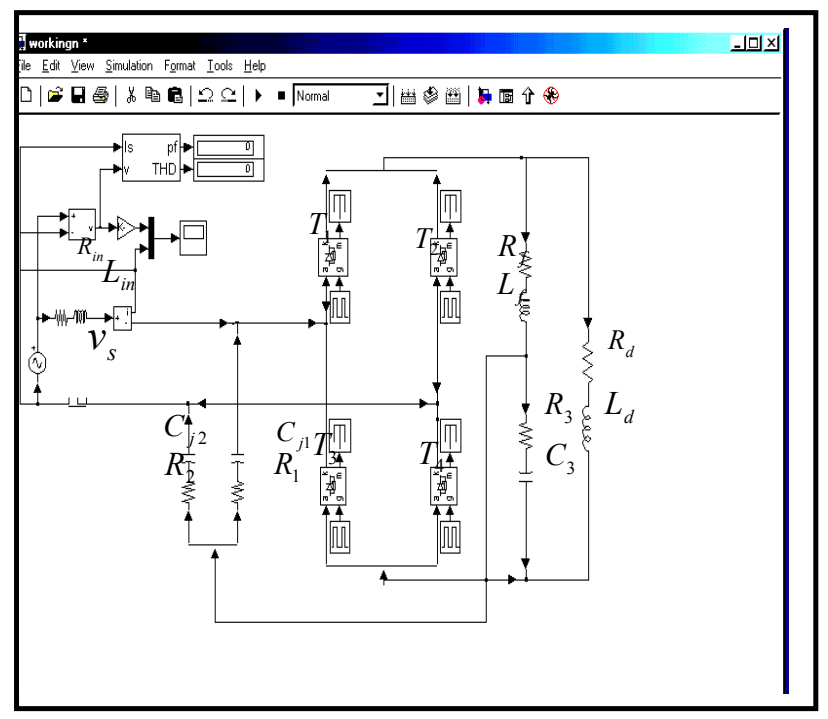

الثكل (9) تمثيل دائرة المقوم القنطري احادي الطور المحكوم مع شبكة الحقن

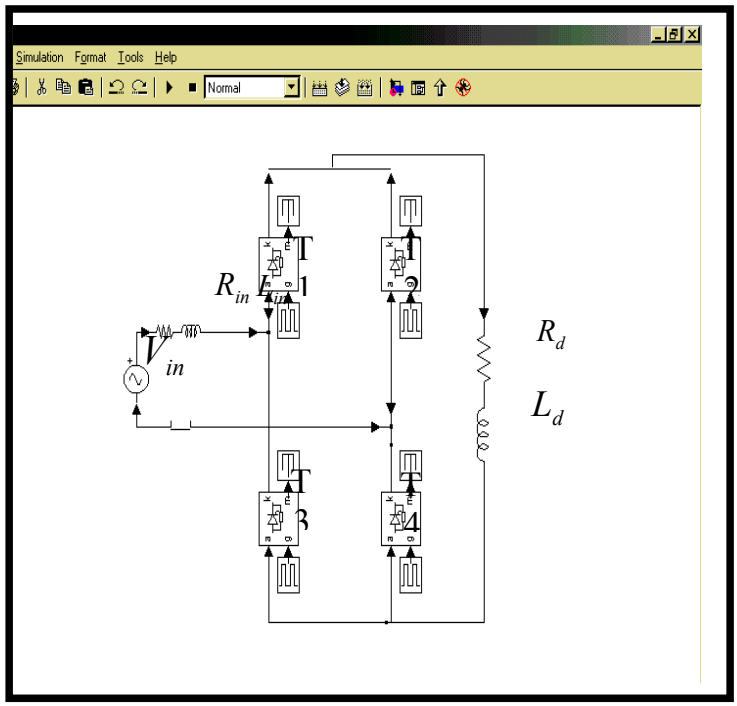

الثكل (8) نمثيل دائرة المقوم القنطري أحادي الطور

جدول (1) نتائج التمثيل للمقوم القنطري أحادي الطور

\begin{tabular}{|c|c|c|c|c|c|c|c|c|}
\hline \multicolumn{4}{|c|}{ بعد ربط شبكة الحقن } & \multicolumn{4}{|c|}{ قبل ربط شبكة الحقن } & \multirow{2}{*}{ زالقدح) زماية } \\
\hline UUF\% & كفاءة المقوم & THD\% & $\overline{\mathrm{PF}}$ & "UF\% & كفاءة المقوم & THD\% & $\overline{\mathrm{PF}}$ & \\
\hline 60 & 92 & 10 & 0.99 & 62 & 98.75 & 48.12 & 0.91 & 0 \\
\hline 57 & 86 & 12 & 0.9 & 55 & 87.4 & 47.2 & 0.8 & 30 \\
\hline 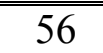 & 82.4 & 14.7 & 0.85 & $\overline{52}$ & 86.5 & 44 & 0.66 & 45 \\
\hline 45 & 80.5 & 21 & 0.72 & 35 & 84.32 & 41 & 0.463 & 60 \\
\hline
\end{tabular}

عند ملاحظة الجدول (1) يمكن استنتاج ما يلي:



هنالك تحسن و اضح في قيمة في فيدة عامل القدرة.

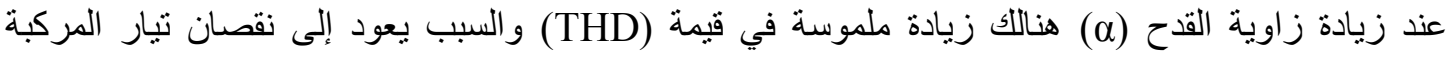
الأساسية بنسبة |cos 2 وليس بسبب ازدياد قيمة النتو افقيات.

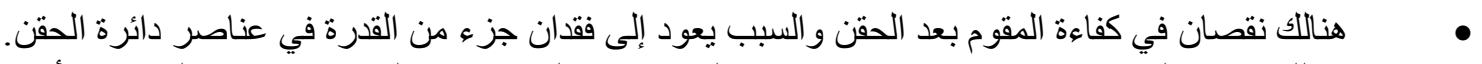

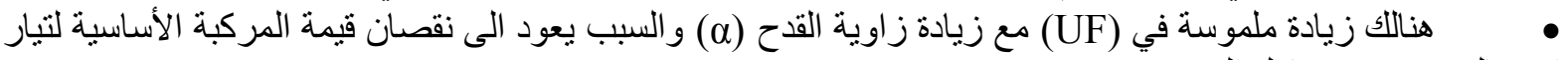

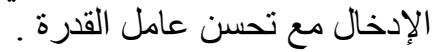



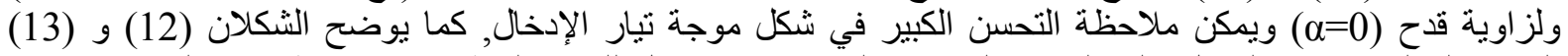


و عند زاوية القدح (a=30) ويمكن ملاحظة التحسن في شكل موجة التيار بعد الحق. أما الشكل (14) يمثل موجة التيار 


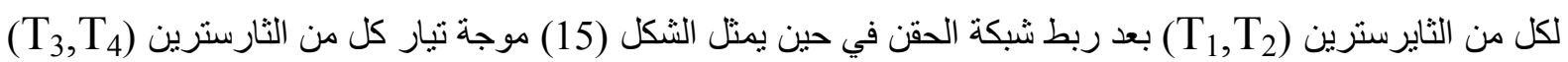

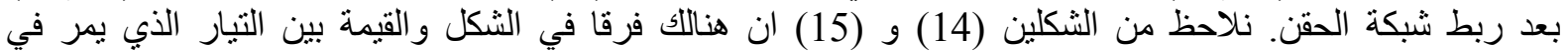

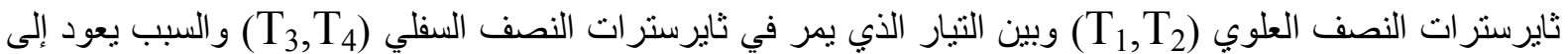

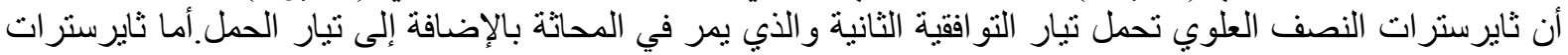
النصف السفلي فتحمل تبار الحمل و العمل

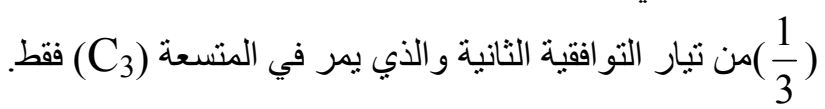
يوضح الثكل (16) موجة التبار لمتسعتي الحقن (الحالة العملية وحالة التمثيل) علما بان هذا التيار يتكون من مركبتين هما

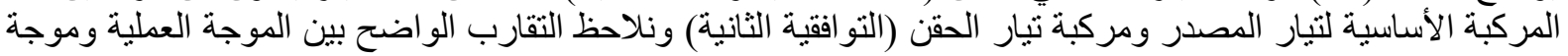


فقط(جزء من تبار الحقن).

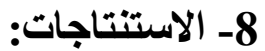

بالاعتماد على نتائج التحليل الرياضي التئي والتمثيل على الحاسبة و النتائج العملية تم التوصل إلى الاستتناجات الآتية:

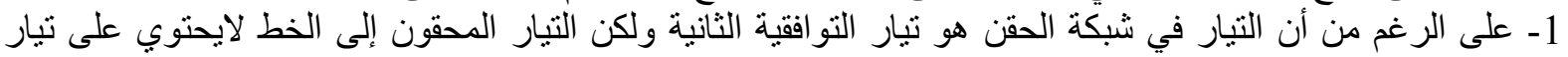

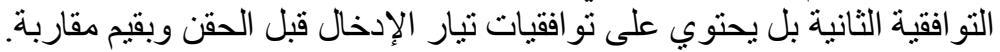
2- عند التحليل النظري لموجة التيار قبل الحقن وبعده وجد بان قيمة تشوه التوافقية الكلي (THD) انخفضت من (43\% إلى 10\%). 3- عند تمثيل دائرة المقوم باستخدام (Matlab-PSB) وجد بان (THD) وبان قيمة تشوه التوافقية الكلي (THD) قبل ربط شبكة الحقن (41,44,47.2,48.12) \% ولزو ايا قدح (60,45,30,0) على الترتيب و عند ربط شبكة الحقن وللقيم نفسها من زو اياي القدح انخفضت قيمة (THD) إلى (21,16,12,10)\% على الترتيب و هذا يدل على فعالية شبكة الحقن في تقليل التو افقيات ومن ثمة تحسين شكل موجة تبار الإدخال.



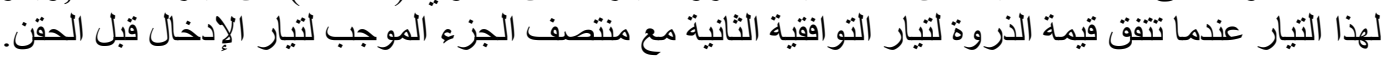

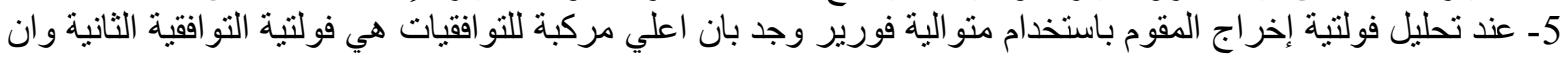

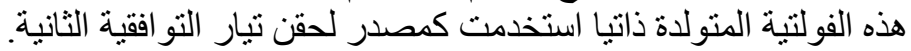

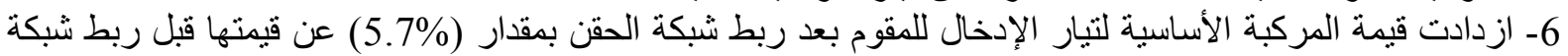

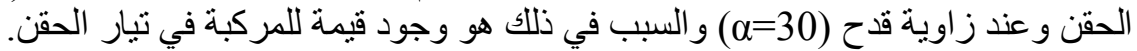

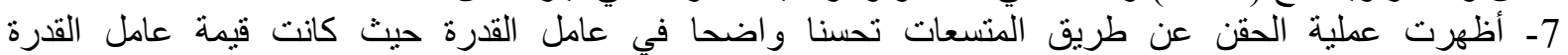

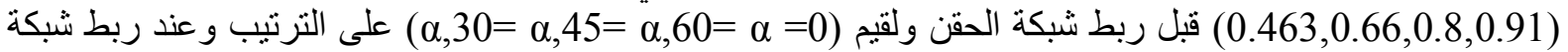
الحقن كان هنالك تحسنا واضحا في عامل القدرة حيث أصبحت قيمته تساوي (0.72,0.85,0.9,0.99) على التربئ

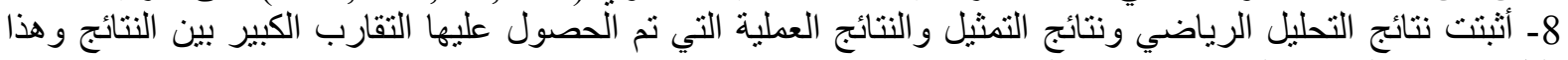

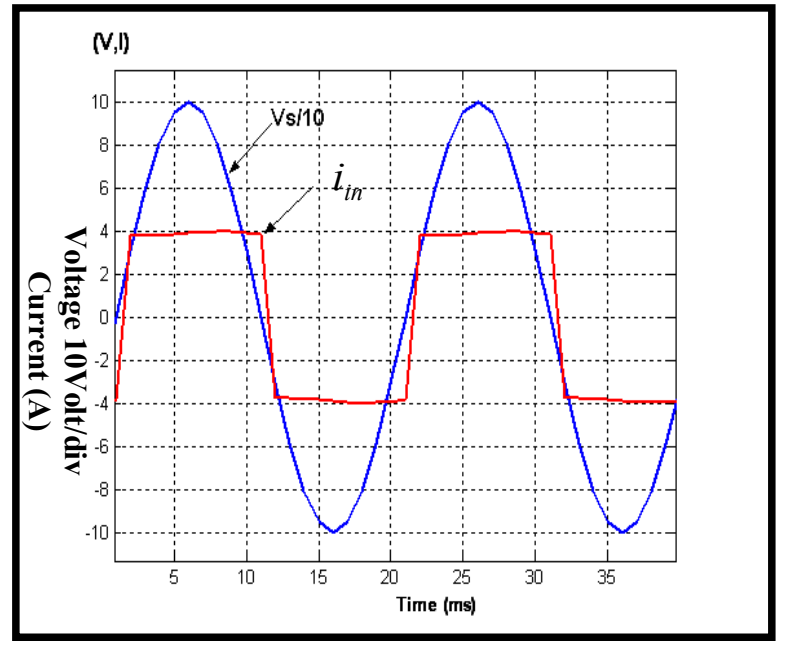

الثكل(10):

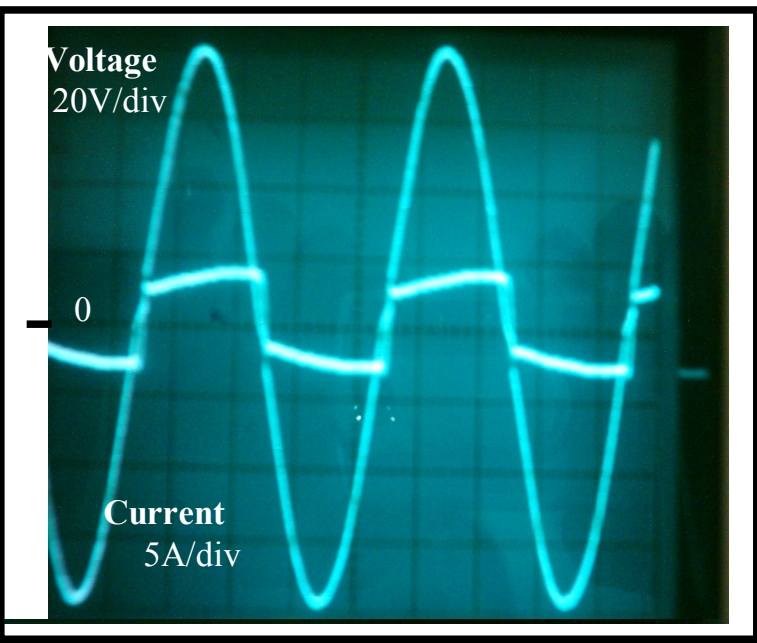

Time $=5 \mathrm{~ms} / \mathrm{div}$

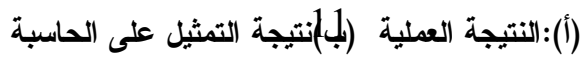




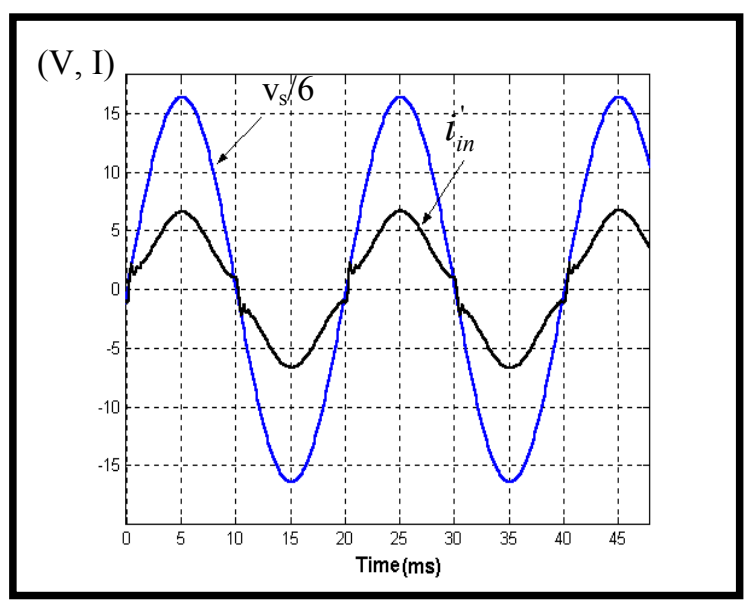

(ب)

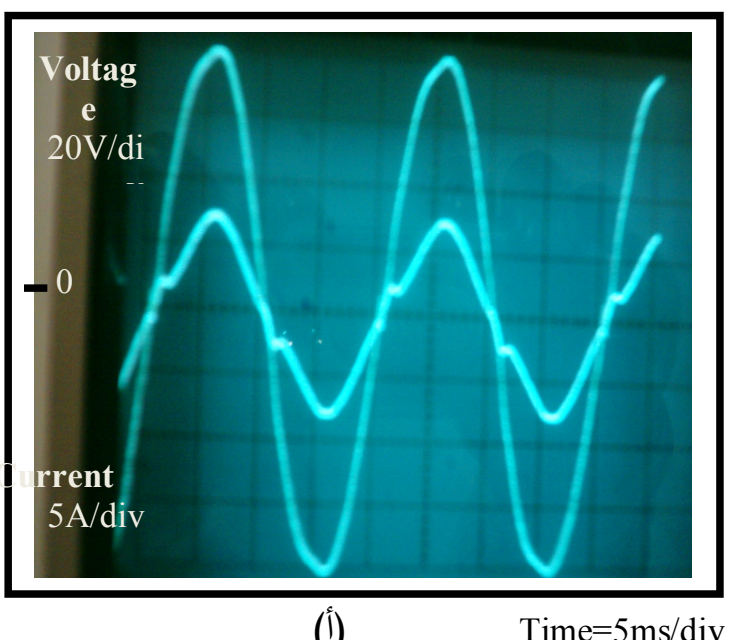

(ا)

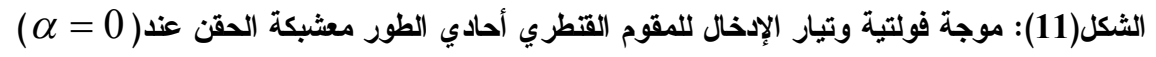

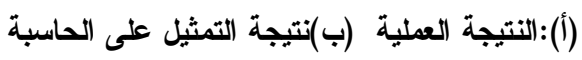

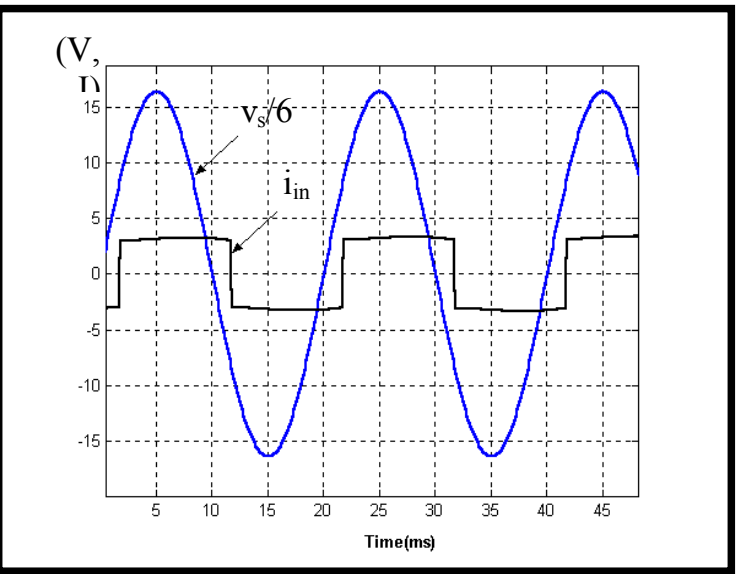

(ب)

الثكل(12): موجة فولتية وتيار الإدخال للمقوم القنطري أحادي الطور بدون شبكة

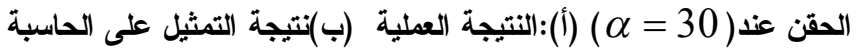

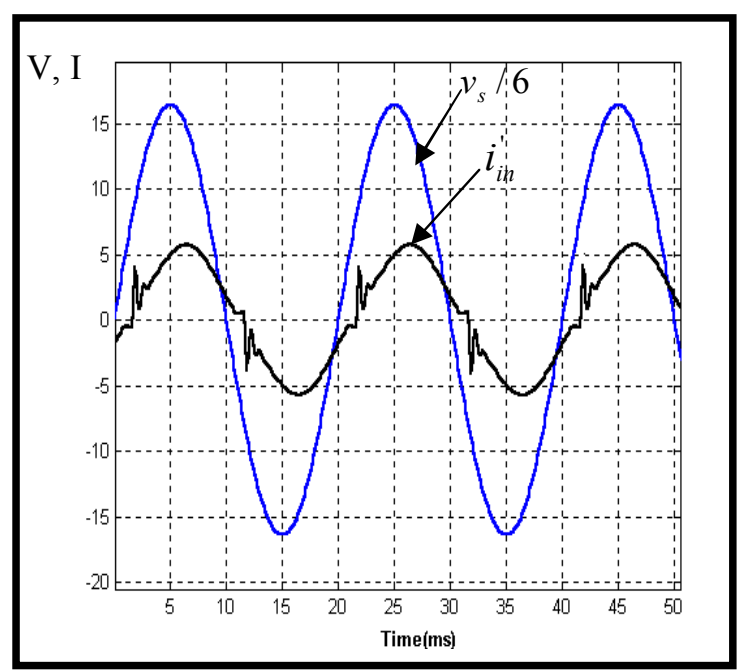

(ب)

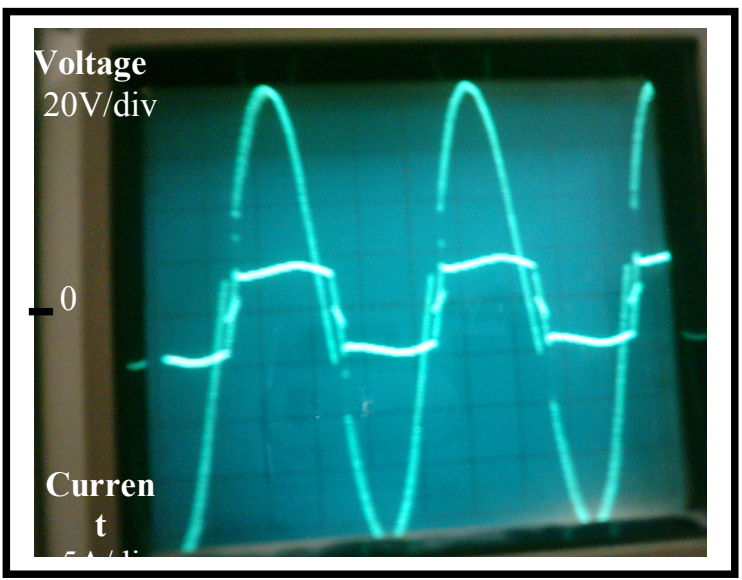

Time $=5 \mathrm{~ms} / \mathrm{div}$

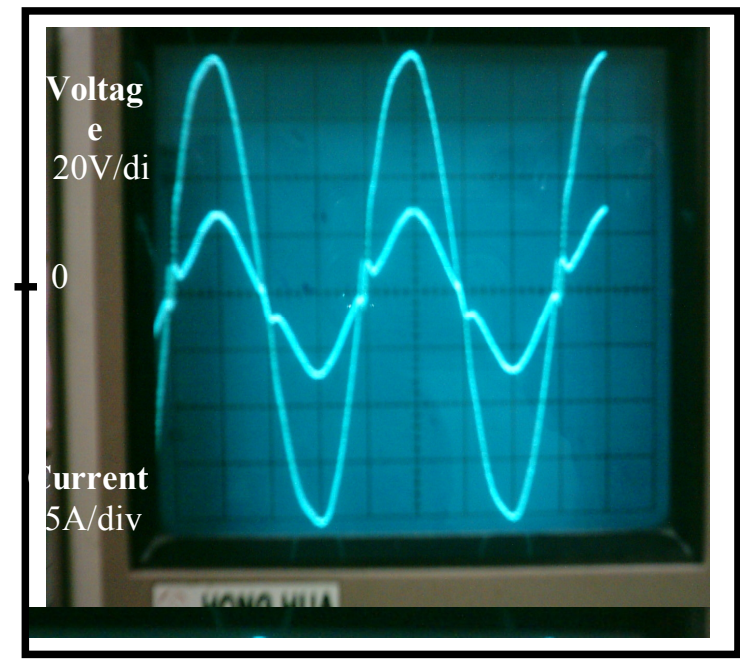

(أ)

Time $=5 \mathrm{~ms} / \mathrm{div}$

الشكل(13): موجة فولتية وتيار الإدخال للمقوم القتطري أحاديالطور مع شبكة الحقن

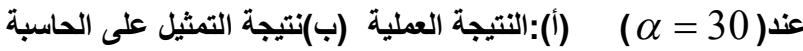


سعيد : تقليل توافقيات التيار مع تحسين عامل القدرة للمقومات أحادية الطور المحكومة بتقنية حقن توافقيات التيار



$(ب)$

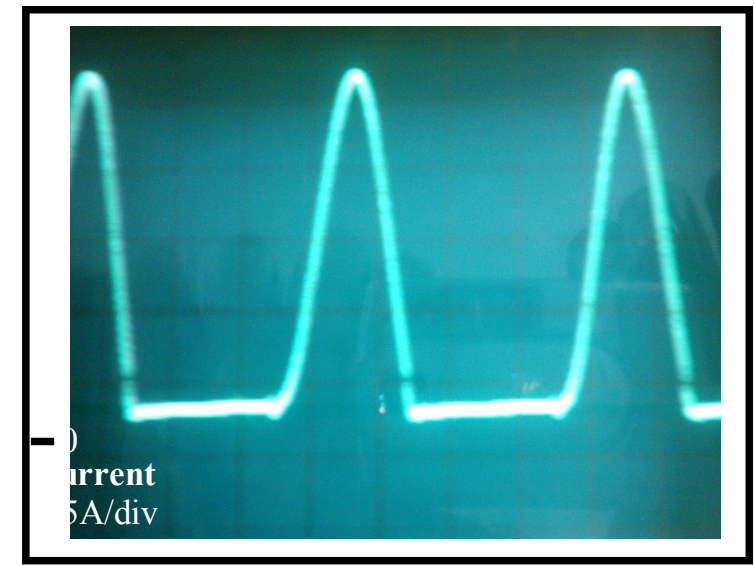

(أ)

Time $=5 \mathrm{~ms} / \mathrm{div}$

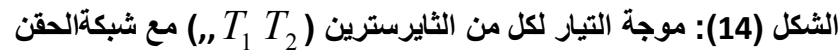

عثد (a=0) (أ):النتيجة العملية (ب):نتيجة التمثيل على الحاسبة



$(ب)$

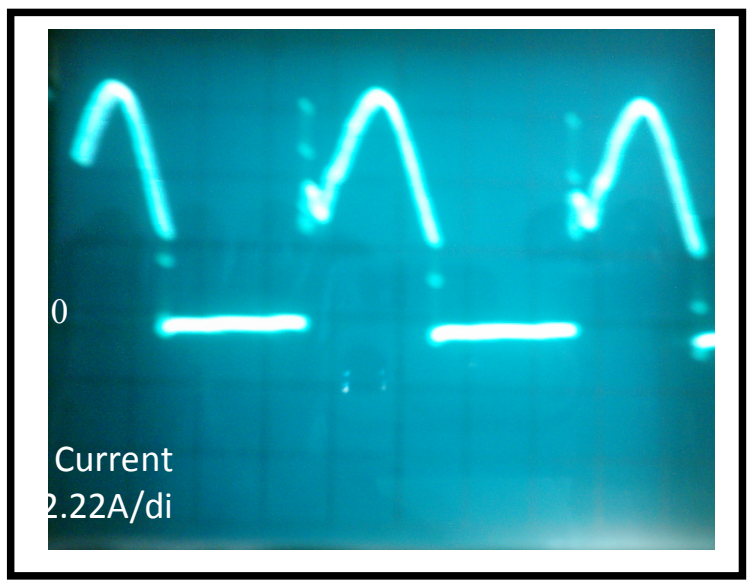

(أ)

Time $=5 \mathrm{~ms} / \mathrm{div}$

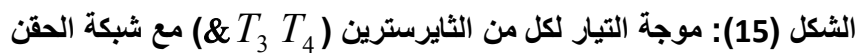



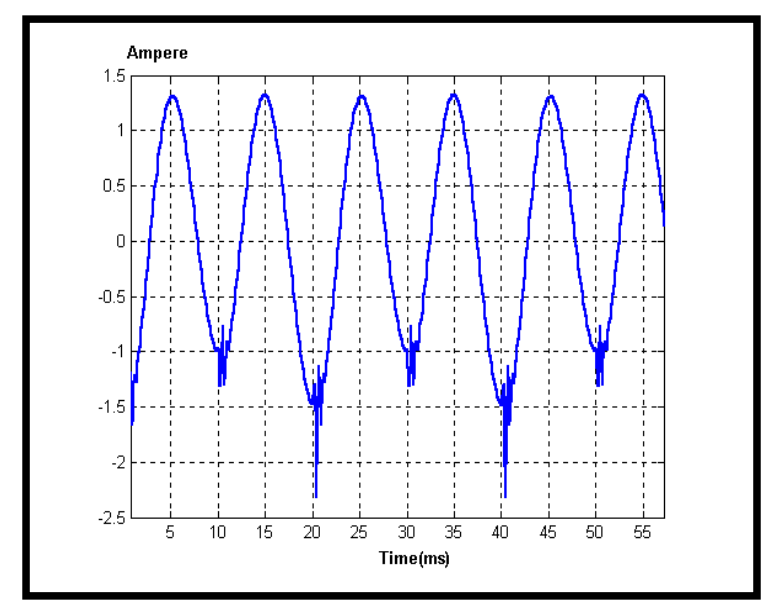

$(ب)$

الثكل (16): موجة التيار لمتسعتي الحقن () (1) (1)



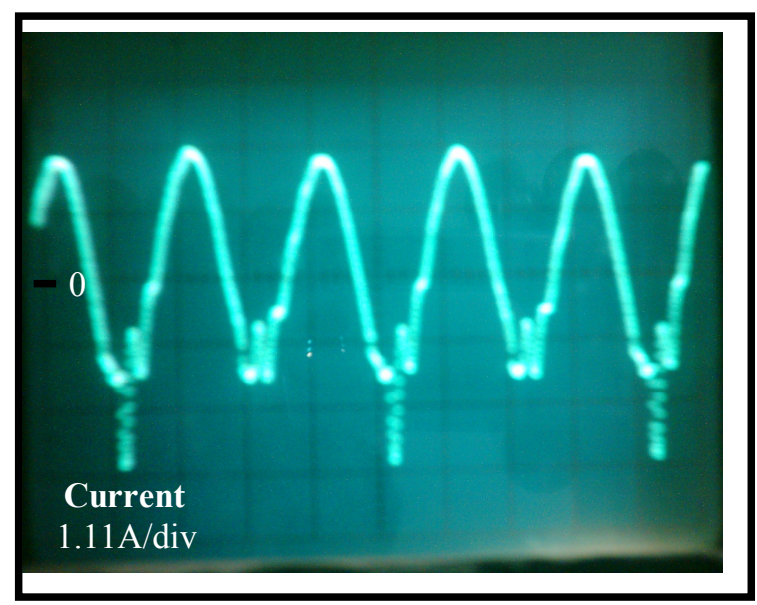

(ا)

Time $=5 \mathrm{~ms} / \mathrm{div}$ 


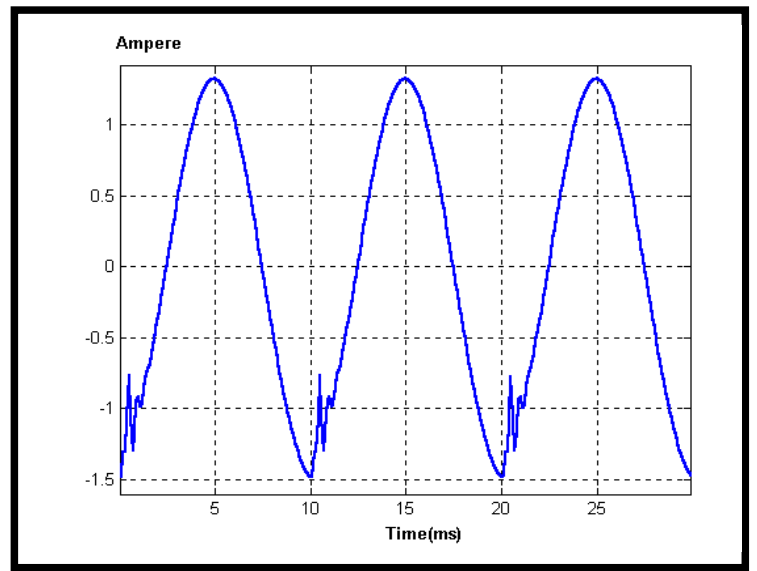

$(ب)$

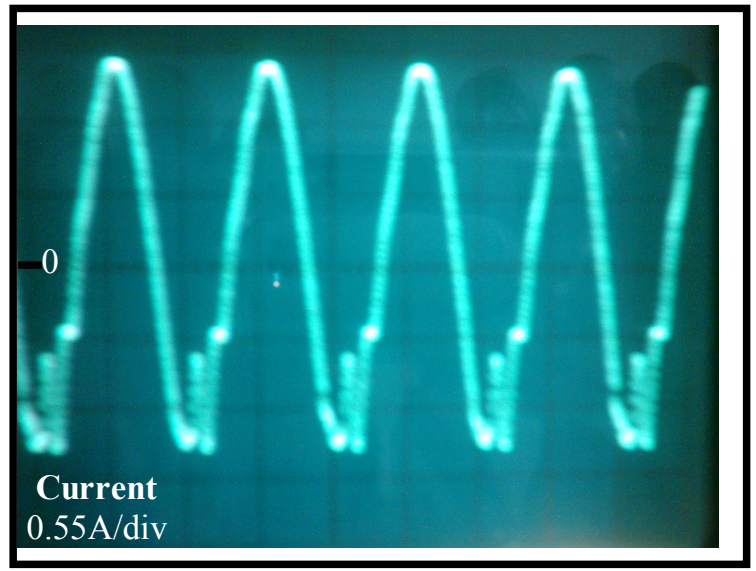

(') Time $=5 \mathrm{~ms} / \mathrm{div}$

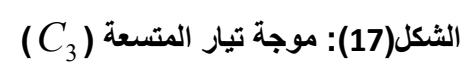

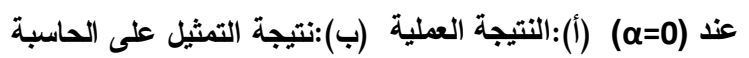

[1] Atluri Rama Prasad, Phoivos D.Ziogas, and Stefanos Manias "A novel Passive Waveshaping Method for Single Phase Diode Rectifiers ",IEEE Transactions on Industrial Electronics, Vol. 37, No.6 ,December 1990.

[2] S.Kim, P.Enjeti. P.Packebush and I.J.Pital,“A New Approach to Improve Power Factor and Reduce Harmonics in A Three Phase Diode Rectifier Type Utility Interface” , IEEE Trans. on Industry Application, Vol.30, No.6 PP.1557-1564, November/December 1994.

[3] X.Dai. , Y.Xu. and D.He. "Novel Passive Rectifier with Low THD Based on Harmonics Injection and Counteracting Principle" ,IEE Trans. on Power Elect. Power Appl., Vol.145, No.4, July1998.

[4 ] C. Sharmeela*, M.R.Mohan, and G.Uma\#"Line Harmonics Reductions Using Neural Based Controller for Shunt Active Filters" ,College of Engineering, Guindy Anna University,Chennai-600 025, India *hellosharmeela@yahoo.co.in, \#uma@annauniv.edu ,March 2003.

[5] A.A.Al-Hyali "AC Current Harmonic Reduction for Single Phase Rectifier Using Current Injection", M.Sc. Thesis , Mosul University ,2006.

[6] Basil M.Saied and Hussein I.Zynal "Minimizing Current Distortion of a Three Phase Bridge Rectifier Based on Line Injection Technique ,IEEE Transaction on Power Electronics, November 2006.

[7] I. Ashida, J.Itoh,"A Novel Three Phase PFC Rectifier Using a Harmonic current Injection Method ", Nagaoka University of Technology, Niigata , Japan,IEEE,2007. 
ملحق) ملاع (A)

قو انين حساب عامل القدرة و الكفاءة و عامل الانتفاع للمحولة

$$
\begin{aligned}
& P_{i n(\text { real })}=\frac{1}{T} \int_{0}^{T}\left(i_{i n} * v_{s}\right) d t \\
& P_{\text {in (app) }}=I_{T(r m s)} * V_{r m s} \\
& P F=\frac{P_{i n(r e a l)}}{P_{i n(a p p)}}=\frac{I_{1} V_{1 r m s} \cos \phi}{I_{T(r m s)} V_{r m s}}=\frac{I_{1}}{I_{T(r m s)}} \cos \phi=K_{\text {dist. }} * K_{\text {disp. }} \\
& \eta=\frac{P_{\text {odc }}}{P_{\text {in (real })}} \\
& P_{o d c}=\frac{1}{T} \int_{0}^{T}\left(i_{L}(t) * v_{L}(t)\right) d t \\
& P U=\frac{P_{o d c}}{P_{i n(a p p)}}
\end{aligned}
$$

على اعتبار أن (

حيث أن:

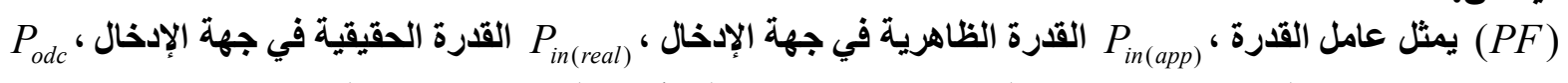

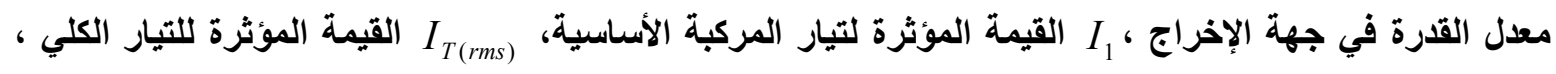

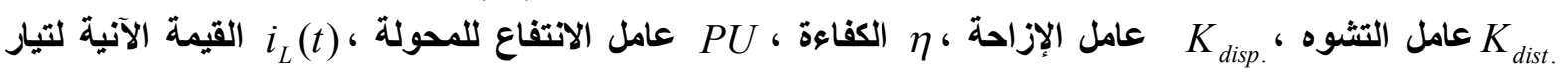
الحمل ، (t) القيمة الآنية لفولتية الحمل. 